\title{
Four pathways involving innate immunity in the pathogenesis of preeclampsia
}

\author{
Kelsey R. Bounds ${ }^{1}$, M. Karen Newell-Rogers ${ }^{2}$ and Brett M. Mitchell ${ }^{1 \text { * }}$ \\ ${ }^{1}$ Department of Medical Physiology, Texas A\&M Health Science Center, Temple, TX, USA, ${ }^{2}$ Department of Surgery, Texas \\ A\&M Health Science Center, Temple, TX, USA
}

OPEN ACCESS

Edited by:

Ulrike Muscha Steckelings, University of Southern Denmark,

Denmark

Reviewed by:

Michael J. Ryan,

University of Mississippi Medical

Center, USA

Styliani Goulopoulou,

University of North Texas Health

Science Center, USA

${ }^{*}$ Correspondence:

Brett M. Mitchell,

Department of Medical Physiology,

Texas A\&M Health Science Center,

702 SW HK Dodgen Loop,

Temple, TX 76504, USA

bmitchell@tamhsc.edu

Specialty section:

This article was submitted to Hypertension, a section of the journal Frontiers in Cardiovascular Medicine

Received: 17 February 2015 Accepted: 14 April 2015

Published: 28 April 2015

Citation:

Bounds KR, Newell-Rogers MK and

Mitchell BM (2015) Four pathways

involving innate immunity in the pathogenesis of preeclampsia. Front.

Cardiovasc. Med. 2:20.

doi: 10.3389/fcrm.2015.00020
The maternal innate immune system plays an important role both in normal pregnancy as well as hypertensive disorders of pregnancy including preeclampsia (PE). We propose four pathways that involve excessive innate immunity that lead to most forms of PE. Pre-existing endothelial dysfunction plus pregnancy leads to an excessive innate immune response resulting in widespread inflammation, placental and renal dysfunction, vasoconstriction, and PE. Placental dysfunction due to shallow trophoblast invasion, inadequate spiral artery remodeling, and/or low placental perfusion initiates an innate immune response leading to excessive inflammation, endothelial and renal dysfunction, and PE. A heightened innate immune system due to pre-existing or acquired infections plus the presence of a paternally derived placenta and semi-allogeneic fetus cause an excessive innate immune response which manifests as PE. Lastly, an abnormal and excessive maternal immune response to pregnancy leads to widespread inflammation, organ dysfunction, and PE. We discuss the potential role of innate immunity in each of these scenarios, as well as the overlap, and how targeting the innate immune system might lead to therapies for the treatment of PE.

Keywords: innate immunity, preeclampsia, endothelial dysfunction, placental dysfunction, immune cells

\section{Preeclampsia and Innate Immunity}

Preeclampsia (PE) is a syndrome in which hypertension and proteinuria or end-organ damage develops during pregnancy. $\mathrm{PE}$, which affects $5-8 \%$ of all pregnancies, usually manifests as early onset ( $<34$ weeks) or late onset ( $\geq 34$ weeks) and is the leading cause of preterm births. While PE typically resolves post-partum, there is a strong connection between $\mathrm{PE}$ and an increased risk of developing cardiovascular disease later in life. In pretty much all studies of women with PE, there are increased levels of pro-inflammatory immune cells and cytokines, decreased levels of regulatory immune cells and cytokines, and/or the ratio of pro-inflammatory to anti-inflammatory immune cells and cytokines is increased. This strongly implicates the maternal immune system as a major contributor to the pathogenesis of PE; however, whether excessive activation of the maternal immune system initiates the development of PE or participates at a later stage in PE or both is unclear.

The maternal innate immune system acts as both a protector and effector during pregnancy. As protector, innate immune cells including macrophages, dendritic cells, natural killer (NK) cells, neutrophils, and $\gamma \delta \mathrm{T}$ cells are upregulated during pregnancy in order to protect the mother from pathogens while her adaptive immune system is dampened so as not to elicit a specific immune response toward the fetus. As effector during normal pregnancy, innate immune cells are important in blastocyst implantation, placentation, trophoblast invasion, and spiral artery remodeling and 
the cellular regulation and repair of the tissues involved in these processes. They are also involved in fetal tolerance throughout pregnancy as well as parturition. However, innate immune cells can switch from a tolerogenic, anti-inflammatory phenotype to a cytotoxic, pro-inflammatory phenotype upon the sensing of pathogens or endogenous danger signals such as RNA, DNA, heat shock proteins, uric acid, tumor necrosis factor, etc. via their expression of highly conserved pattern recognition receptors. As cytotoxic effector cells, innate immune cells create a state of oxidative stress by releasing reactive oxygen/nitrogen species (ROS/RNS), inflammation via cytokine release and activation of adaptive $T$ and $B$ cells, and placental ischemia via reduced angiogenesis and increased vasoconstriction, in an attempt to cause cell death, fibrosis, and rejection of the fetus. We believe that the timing and level of innate immune system activation during pregnancy corresponds to not only the severity of PE but also pregnancy outcome.

Does excessive activation of the maternal innate immune system initiate the development of $\mathrm{PE}$, participate at a later stage, or both? We believe the answer is both. In this review, we present evidence that excessive innate immunity during pregnancy can lead to the clinical manifestations of PE regardless of the initiating pathophysiology which can be divided into four pathways.

\section{Four Pathways Involving Innate Immunity in the Pathogenesis of Preeclampsia}

Based on known risk factors for the development of PE as well as experimental and circumstantial clinical evidence, we propose four pathways involving excessive innate immune system activation that can lead to PE. Two pathways consist of excessive innate immunity in response to abnormal physiology which in turn leads to inflammation, angiogenic imbalance, endothelial/placental/ renal dysfunction, and PE. The other two pathways consist of heightened or abnormal innate immunity prior to and/or during pregnancy leading to excessive inflammation, angiogenic imbalance, endothelial/placental/renal dysfunction, and PE. The four pathways are illustrated in Figure 1.

\section{Endothelial Dysfunction, Pregnancy, and Innate Immunity}

The first pathway involves pre-existing endothelial dysfunction that, when coupled with the adaptations of pregnancy, is not sufficient to provide blood flow to the appropriate tissues. Pregnancy is considered a physiological stress test and the low-grade inflammation evident in the vasculature of obese women, women $>35$ years of age, women with either diabetes mellitus type I or II (T1DM and T2DM), and hyperlipidemia starts the mother off in a predicament. The increased volume, cellular metabolic activity, and tissue demands of pregnancy all strain the dysfunctional endothelium of the maternal systemic and reproductive vasculature and elicit further damage leading to enhanced activation and recruitment of innate immune cells and an augmented inflammatory immune response.

Obesity is a known risk factor for PE, but the mechanisms are not yet known. Bodnar and colleagues studied the occurrence of PE in women who were divided into groups based on their body mass

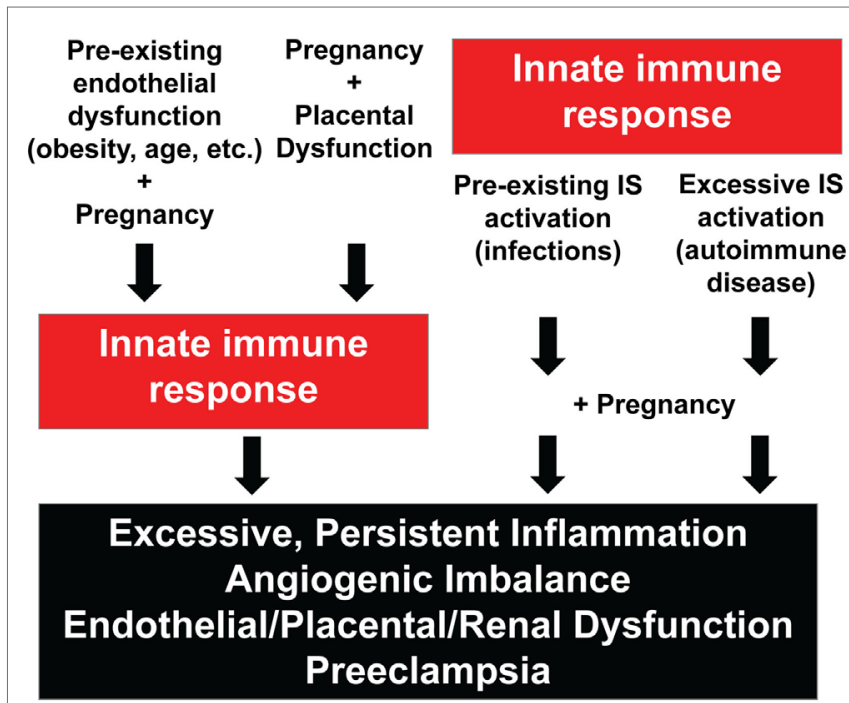

FIGURE 1 | Four pathways involving innate immunity in the pathogenesis of preeclampsia. IS, immune system.

index (BMI) and they found a correlation between high pregnancy BMI and risk for both mild and severe PE (1). This corroborates another study that searched for different risk factors associated with severe PE. Stone et al. retrospectively compared women with $\mathrm{PE}$ versus normotensive pregnant women and found that the only risk factors related to severe PE were a history of the disease and severe maternal obesity (2). A question that arises is whether the pregnant women have a high BMI before becoming pregnant or gained the weight during pregnancy and whether or not that has an impact on the development of PE. Increased pre-pregnancy BMI was found to be significantly associated with an increase in the risk for PE in a population-based cohort study done by Ros and colleagues (3). They found that $9.1 \%$ of overweight (BMI $=26.1-29.0)$ and $12.9 \%$ of obese (BMI $>29.0$ ) women developed PE compared to $3.1 \%$ of underweight (BMI < 19.8) and $4.5 \%$ of normal weight $(\mathrm{BMI}=19.8-26.0)$ women. The women in the obese category had an odds ratio of 5.19 for PE. Another study done by Bianco et al. looked at morbidly obese (BMI > 35) pregnant women and found that these women were more likely to develop PE compared to non-obese women (4). They also reported that gestational weight gain did not affect the risk of developing PE. Saftlas et al. studied the difference between pre-pregnancy BMI and gestational weight gain and whether either one caused an increased risk of developing PE (5). They concluded that women who were considered obese before pregnancy had a higher risk of PE. In contrast, women who gained more weight than expected during pregnancy did not have an increased risk for PE; however, they did have a higher risk of developing transient hypertension. Gestational weight gain does not seem to contribute much to the risk of developing PE compared to pre-pregnancy obesity, as it is not always associated with an increased BMI and thus is not tightly linked to PE (6). Morbid obesity is highly associated with endothelial dysfunction. Mauricio and colleagues stated that there are many contributions to endothelial dysfunction in obese patients, but the most outstanding factors 
included diminished bioavailability of nitric oxide, oxidative stress, chronic inflammation, increased amounts of vasoconstrictors, and decreased amounts of vasodilators in the body (7). Women with a higher BMI begin pregnancy with an increased blood volume, cardiac output, and blood pressure as well as chronic inflammation and endothelial dysfunction which in turn may cause PE when these become exacerbated during pregnancy. We propose that this occurs when the innate immune system is excessively activated by danger signals stemming from the recurring endothelial injury and dysfunction. Innate immune system activation then augments the inflammation, oxidative stress, and vasoconstriction which manifests as PE. Experimental evidence supports this contention as a loss of nitric oxide, vascular endothelial growth factor (VEGF), or transforming growth factor beta (TGF $\beta$ ) bioavailability during pregnancy as well as ingesting a high-fat diet can induce PE-like features in animals (8-11).

Several studies have reported a correlation between obesity and neutrophil infiltration of the systemic vasculature in pregnant women. Neutrophils help protect the host from infection by producing ROS/RNS as well as proteolytic enzymes that are helpful in fighting infection, but that can sometimes be toxic to the host tissue as a part of a "by-stander" type injury. Leik and Walsh reported that neutrophils act as carriers of oxidative stress from the placenta to the maternal vasculature by adhering to the endothelium (12). They biopsied highly vascularized subcutaneous fat at the time of cesarean section from normal pregnant and women with PE and stained for interleukin (IL)-8, intercellular adhesion molecule-1 (ICAM-1), and cluster of differentiation 66b (CD66b). There was a greater amount of staining for IL-8, an inflammatory marker, observed in the vascular smooth muscle and endothelial layers of women with PE. This represented the migration of neutrophils from the circulation to the vascular smooth muscles because neutrophils tend to travel on a concentration gradient toward increasing concentrations of IL-8. ICAM-1 was found on the endothelium in all groups, but only in the vascular smooth muscle layer of the PE group which supports the idea that inflammation affects the vascular smooth muscle cells of women with PE. Levels of CD66b were significantly greater in women with $\mathrm{PE}$ suggesting that there were more neutrophils present. There were also more vessels containing adhered and flattened neutrophils on the endothelium in women with PE. This connection between neutrophils and inflammation could be one of the links between obesity, innate immunity, and PE.

Overweight and obese women are more likely to be diagnosed with chronic disease risk factors such as hypercholesterolemia which further complicates their pregnancies. Elevated levels of triglycerides (TG) and low density lipoproteins (LDL) are present in many women with PE suggesting that women with hyperlipidemia may be at risk for developing PE. The main focus of concern is high levels of TGs in the body. Hubel et al. found that women with PE had significantly higher amounts of TG in their serum, but the high density lipoproteins (HDL) and LDL concentrations did not differ between women with PE and normal pregnant women (13). Sattar et al. produced similar findings and both groups suggest that the oxidation of TGs play a role in the development of endothelial dysfunction in women with PE (14). According to Granger et al., the significant increase in TG in women with PE correlates with an increase in small dense LDLs (15). Fatty acids serve as substrates for lipid peroxidation which is also significantly increased in women with PE. ApoC3 transgenic mice, which exhibit abnormal fatty acid metabolism, display PE-like features (16-18). Oxidation of lipids in the body can directly cause endothelial dysfunction as well as act as danger signals that activate the innate immune system which further worsens endothelial injury. Xu and colleagues found the pro-inflammatory innate immune system receptor TLR4 in lipid-rich, macrophage-infiltrated atherosclerotic areas of humans and apolipoprotein E deficient mice (19). They also discovered that TLR4 is upregulated by oxidized LDL in cultured macrophages. Tuten et al. investigated the relationship between polymorphisms in lectin-like oxidized low-density lipoprotein receptor (LOX-1) genes and circulating sLOX-1 and oxLDL levels and the risk of PE (20). They found that certain polymorphisms of the LOX-1 gene (LOX-1 3'UTR188C>T and K167N) and high plasma levels of sLOX-1 were significantly associated with an increased risk of developing PE. Sankaralingam et al. reported an upregulation of LOX-1 and arginase in the vasculature of women with PE which can contribute to oxidative stress $(21,22)$. The mechanism of how this occurs is still unclear. However, they found that methylglyoxal, which is involved in vascular complications of diabetes mellitus and the development of hypertension, is a possible factor that affects LOX-1 and arginase because it is able to induce oxidative stress in vascular cells. Another finding by Zhang and colleagues suggests that LOX-1 accumulation may contribute to the development of PE by promoting sFlt-1 production in trophoblasts (23). This group was able to inhibit LOX-1 and protect against oxidative stress-mediated trophoblast dysfunction. We believe that hyperlipidemia, excessive lipid oxidation, and endothelial dysfunction coupled with a persistent innate immune response during pregnancy can elicit PE in some women.

A chronic disease that can result from obesity and hyperlipidemia is T2DM. T2DM is characterized by insulin resistance which is usually intensified by pregnancy thus complicating maternal health. An outcome analysis of pregnancies in women with T2DM showed that diabetic women were diagnosed with PE two times more than non-diabetic women (24). Garner et al. performed a prospective controlled study comparing the incidence of PE and maternal-fetal outcome in diabetic pregnancies and non-diabetic pregnancies (25). Diabetic women were 9.9\% more likely to develop PE compared to $4.3 \%$ in non-diabetic pregnant women. PE also became more prevalent with the increasing severity of diabetes. One might ask whether there is a difference between the prevalence of PE in T1DM and T2DM. Cundy et al. compared pregnant women with T1DM and T2DM and found that the incidence of hypertension was similar, but the subgroups of hypertension were different (26). Women with T2DM were more inclined to have chronic hypertension whereas women with T1DM were more frequently diagnosed with PE. Up to $39 \%$ of pregnant women with T1DM are affected by PE. This could be explained by the hyperglycemia causing endothelial dysfunction in the maternal and placental vessels prompting innate immunity and the development of PE $(27,28)$. There are many speculations as to how hyperglycemia affects endothelial cell function, but the ideas that are most prevalent are that hyperglycemia causes the activation of the polyol pathway, the activation of protein kinase $\mathrm{C}$, and 
increases oxidative stress (29). These different pathways seem to overlap in certain aspects so they are considered to work together in some ways to diminish endothelial cell function. Hyperglycemic patients also tend to exhibit increased innate immunity causing inflammation. Liu et al. examined the effects of high glucose on macrophages and found that proliferation increased with greater concentrations of glucose possibly due to increased CSF-1 receptor expression (30). Increased macrophages and macrophage polarization to pro-inflammatory $\mathrm{M} 1$ cells result in inflammation because of their innate immune system activity (31). The deteriorating endothelial dysfunction and innate immune system activation due to hyperglycemia may be sufficient in some women to cause PE.

Advanced maternal age (AMA; 35 years or older) is considered another risk factor for PE. In the United States, the risk of PE increases by $30 \%$ every year beyond 34 (32). Little is known as to why PE occurs more often in older pregnant women but there has been some speculation. Bianco and colleagues found that out of 1,404 pregnant women, older gravidas were more likely to develop $\mathrm{PE}$ which correlated with other serious pregnancy related diseases (33). Lee et al. studied the prevalence of PE in an Asian population and found that women above the age of 34 years were more likely to develop PE (34). Dorjgochoo and colleagues measured in vivo lipid peroxidation and found that levels of a biomarker significantly increased with age in middle-aged and postmenopausal women (35). They also found that a marker for oxidative stress was positively associated with age. Csiszar and colleagues suggest that age-related oxidative stress may promote vascular inflammation and endothelial dysfunction (36). Sorescu et al. found that increased expression of NOX4/NADPH oxidase (ROS producing enzyme) correlated positively with vascular superoxide production and atherosclerosis and inflammation in aging humans (37). There is also emerging data suggesting that the innate immune system is upregulated during aging (38). This supports the notion that the innate immune system is involved in inflammation, endothelial dysfunction, and PE during AMA pregnancy.

\section{Pregnancy, Placental Dysfunction, and Innate Immunity}

The second pathway involves placental dysfunction leading to innate immune system activation and resulting in inflammation, endothelial/renal dysfunction, and PE. Danger signals including RNA, DNA, heat shock proteins, uric acid, tumor necrosis factor, and others released from the placenta "tell" the mother that the placenta either did not form properly or is not functioning adequately (39). This would lead to fetal rejection as the mother attempts to terminate the pregnancy and save herself. As in solid organ transplant rejection, the result of innate immune system activation toward an organ results in innate and adaptive immune cell infiltration, inflammation, decreased angiogenesis, and reduced perfusion in an effort to cause ischemia, fibrosis, and cell death. It has been suggested that the severity of PE is associated with how strong or weak the innate immune response is.

Placental dysfunction including shallow trophoblast invasion, deficient spiral artery remodeling, and low placental perfusion are known to be involved in the development of PE; however, the root of this problem is still unknown $(40,41)$. In normal pregnancy, blastocysts latch on to the maternal decidua then the cytotrophoblast cells (CTBs) proliferate and create extravillous trophoblasts on the very tip of their columns. These cells invade the decidua and differentiate into either endovascular or interstitial trophoblasts. While the interstitial trophoblasts embed themselves into the inner myometrium, the endovascular trophoblasts, derived from the male, start migrating toward the maternal spiral arteries. At around 10-12 weeks of gestation in women the trophoblasts break down the maternal vessel walls which create low-resistance vessels to maintain sufficient placental perfusion. When this process does not progress successfully, PE has been shown to develop. This means that the maternal innate immune system has to allow the perfect interaction between the paternally derived trophoblasts and the maternal tissue for proper invasion. Different studies have looked into the cause of trophoblast invasion failure.

Zhou et al. suggests that in normal pregnancies, in order for the CTBs to invade the uterine interstitium and vasculature the CTBs have to alter their adhesion receptor phenotype to model the maternal endothelial cells they are to replace (42). In PE pregnancies this modification is thought to not occur. CTBs from normal pregnant women and CTBs from women with PE were examined for whether or not they expressed vascular cell adhesion molecule (VCAM-1) and platelet-endothelial cell adhesion molecule (PECAM-1) along with other integrins and cadherins that are characteristic of endothelial cells and certain leukocytes. They found that VCAM-1 was not found on the villous CTBs but on the CTBs within the uterine wall of normal pregnant women. PECAM-1 was expressed on the interstitial and endovascular CTBs in normal pregnant women; however, neither VCAM-1 nor PECAM-1 were found on CTBs in women with PE. Coukos et al. reported that PECAM-1 is present in the trophoblast-endothelium interaction which suggests that PECAM-1 is an important part of proper trophoblast invasion found in normal pregnancies (43). They found that certain trophoblasts express PECAM-1, suggesting that there is a subpopulation that is required for the endovascular differentiation pathway.

If this trophoblast invasion does not occur properly then danger signals are released from the placenta which are detected by the local and maternal systemic innate immune system and elicit a response from innate immune cells including macrophages, dendritic cells, NK cells, neutrophils, and $\gamma \delta T$ cells (39). Once these cells are activated excessive inflammation leads to endothelial dysfunction and PE. We and others have reported that the innate immune system receptors TLR3, TLR4, TLR7, and TLR8 are increased significantly in placentas of women with PE $(44,45)$. Additionally, TLR9 activation by mitochondrial DNA/fetal DNA or their mimetics during pregnancy in rodents can also cause detrimental pregnancy outcomes $(46,47)$. This supports the notion that when trophoblast invasion fails danger signals are released that activate the innate immune system.

If placental trophoblasts invade correctly but the maternal spiral arteries do not remodel in a sufficient manner there is also a chance of developing PE. Normal pregnancies are characterized by the formation of large arterio-venous shunts whereas during PE there are minimal shunts resulting in narrower uterine arteries (48). For a normal pregnancy to occur, it is essential that the spiral arteries adapt 
to the placental invasion by dilating the distal segment to ensure the delivery of large amounts of blood to the placenta at an appropriate rate and pressure. When there is improper remodeling the spiral arteries are constricted which results in faster output of blood causing the rupturing of anchoring villi as well as the dislodging of trophoblastic micro-particulate debris from the villous surface leading to maternal endothelial and immune cell activation. Finally, a reduction in blood transit time impairs oxygen exchange. During normal spiral artery remodeling, the surrounding smooth muscle loses its elasticity, but in PE the smooth muscle constricts thus reducing blood supply to the placenta. Singh and colleagues reported that pregnant mice lacking complement component $\mathrm{Clq}$, necessary for trophoblast invasion and spiral artery remodeling, exhibit many features of $\mathrm{PE}$ supporting a role for innate immunity in placentation that, when deficient, can lead to low placental perfusion, innate immune system activation, inflammation, angiogenic imbalance, endothelial/renal dysfunction, and PE (49). In severe cases, the immunity, inflammation, angiogenic imbalance, and endothelial dysfunction can cause fibrosis of the uterine wall leading to fetal rejection.

\section{Infections, Innate Immunity, and Pregnancy}

The third pathway involves innate immune system activation in response to infections acquired before or during pregnancy coupled with the higher sensitivity of the innate immune system during pregnancy. We propose that this "double hit" immune response exceeds a certain threshold in some women and the excessive pro-inflammatory and oxidative state then affects the vasculature, placenta, and kidneys. It has been reported that bacterial and viral infections significantly increase the risk of developing PE (50). Chronic subclinical infections are suggested to be a probable cause of inflammation in women that develop PE and that early treatment of vaginal and urinary infections reduces the incidence of developing PE (51). Another study corroborated these findings reporting that not only urinary tract infections are associated with an increased risk of PE, but also periodontal infections as well (52).

Highly conserved pattern recognition receptors including TLRs, NOD-like receptors (NLRs), and RIG-like receptors (RLRs) detect various parts of bacteria and upon receptor activation induce an innate immune response. For TLRs, bacterial lipoproteins are detected by TLR1, bacterial peptidoclycans and lipoteichoic acid of Gram-positive bacteria are detected by TLR2, heat shock proteins and lipopolysaccharide of Gram-negative bacteria are detected by TLR4, bacterial flagellin is detected by TLR5, mycoplasma is detected by TLR6, unmethylated CpG DNA is detected by TLR9, and a specific bacterial ribosomal RNA sequence is detected by TLR13, all of which reside on the surface of maternal immune and non-immune cells as well as cells in the uteroplacental unit. Activation of these TLRs leads to a rapid anti-bacterial immune response for pathogen clearance followed by adaptive immune system activation. While direct cause-and-effect studies in women showing that bacterial infections induce PE are obviously lacking, there is evidence that bacterial infections are highly associated with the development of PE and experimentally can elicit PE in rodents.

Amarasekara and colleagues reported that $12.7 \%$ of placentas from women with PE were positive for bacteria whereas all placentas from normotensive women were negative (53). Bacteria found to be present in the placentas of women with PE included Bacillus cereus, Listeria, Salmonella, Escherichia, Klebsiella pneumonia, Anoxybacillus, Variovorax, Prevotella, Porphyromonas, and Dialister. Periodontal infections from Porphyromonas gingivalis, Eikenella corrodens, and Micromonas micros were also found more often in women with PE $(51,54)$. Additionally, placental TLR4 expression is increased in women with PE compared to normotensive women $(55,56)$. While not all studies report a significant association between bacterial infection and PE, many do and further suggest that inflammation from chronic subclinical infection rather than acute infection or reinfection is associated with the development of PE (50). Experimentally, endotoxin/LPS given to pregnant rats and mice were able to induce PE-like features by inducing excessive inflammation $(16,57)$. Additionally, TLR9 activation by $\mathrm{CpG}$ oligonucleotides during pregnancy in rats can also elicit PE-like features (47). While these animal studies examined the effects of acute bacterial infection on pregnancy, studies in which animals are chronically infected prior to pregnancy are needed. Overall, these findings support the notion that bacterial infection and subsequent innate immune receptor activation may lead to excessive inflammation and PE in some women.

Other TLRs, NLRs, and RLRs detect viruses and upon ligation also induce an innate immune response. For TLRs, maternal and uteroplacental cells contain TLR3 which recognizes dsRNA and TLR7/8 which recognizes ssRNA in addition to TLR9 and TLR13 which also recognize patterns of DNA viruses. Activation of these TLRs induces a rapid anti-viral immune response mainly mediated by type I interferons and also leads to adaptive immune system activation. Like bacterial infections, there is circumstantial evidence associating viral infections and $\mathrm{PE}$. These include adeno-associatedvirus-2, parvovirus, cytomegalovirus, and herpes simplex virus. Other viruses that may be associated with PE include rhinovirus, reovirus, enterovirus, coxsackievirus, rhabdovirus, paramyxovirus, orthomyxovirus, and picornavirus. We have reported that TLR3, TLR7, and TLR8 immunoreactivity is increased in placentas of women with PE compared to normotensive women along with markedly increased staining for placental dsRNA (44). We have also discovered that maternal innate immune system activation using TLR3 or TLR7/8 agonists induces PE-like features in rats and mice and that treatment with the immunoregulatory cytokines IL-10 and IL- 4 can prevent these effects $(44,58-62)$. Supportive of this innate immunity-PE pathway is that the induction of PE-like features by viral mimetics in rodents is pregnancy-dependent and is associated with increased pro-inflammatory immune cells and cytokines and excessive inflammation. As mentioned previously, TLR9 activation also induces PE-like features in rats (47). Future studies examining whether these various pathogens and others can directly induce PE in animals as well as testing for these pathogens early in pregnant women will help define the connection between infections and the development of PE.

\section{Abnormal Innate Immunity to Pregnancy}

The fourth pathway involves an exaggerated maternal immune activation relative to a normal pregnancy. Supportive of this mechanism are the findings that women with a personal or familial 
history of autoimmune disease are at a significantly higher risk of developing PE. Each person has individualized immune responses as determined by the amount and type of major histocompatibility complex (MHC) molecules expressed on their cells. Women with a hyperactive innate immune system would initiate an exaggerated immune response to the cellular necrosis that occurs during implantation and placentation as well as to the fetal and paternal antigens. This typically manifests not only as excessive inflammation but also eventually by the presence of autoantibodies against various proteins contributing to the development of PE.

Classical studies showing that reduced exposure to paternal antigens prior to pregnancy or in vitro fertilization increases the risk of PE support the notion that maternal tolerance to the fetus is necessary for a normotensive, successful pregnancy. Maternal and placental immune cells receiving signals released from the fetoplacental unit usually achieve this state of tolerance; however, if maternal innate immune cells "over-ride" these signals and do not lead to a state conducive to tolerance, then excessive innate immune system activation and inflammation occur. This is what likely happens in women with autoimmune diseases. Women with systemic lupus erythematosus (SLE), rheumatoid arthritis (RA), antiphospholipid syndrome, autoimmune thyroid disease, and T1DM all have an increased risk of developing PE $(25,63,64)$. The increased innate immunity in these women could be due to altered complement regulatory proteins or in the expression of placental sialic acid $(64,65)$. Some studies have identified that the KIR AA genotype of uterine NK cells inhibits fetal HLA-C2 expressed on trophoblasts, while genetic variants in fetal HLA-G do not dampen maternal immune activation that is associated with PE $(66,67)$. Lastly, decreased levels of inducible regulatory $\mathrm{T}$ cells (iTregs) or the ratio of iTregs to pro-inflammatory $\mathrm{T}$ cells have been reported to be decreased in women with autoimmune diseases as well as in women with PE (41, 68-72). iTregs are known to reduce antigen-specific immune cell activation and signaling. Hsu and colleagues reported that antigen presenting cells from the decidua of normal pregnant women greatly promote the expansion of iTregs whereas antigen presenting cells from the decidua of women with PE do not (68). This lack of regulatory control of immune activation may contribute in some women to the excessive inflammation and organ dysfunction that leads to PE.

\section{Links Between Innate Immunity and the Manifestation of Preeclampsia}

Regardless of the pathway involved, excessive activation of the innate immune system may ultimately lead to the features of PE including hypertension, proteinuria, and end-organ damage. This is likely mediated by both the innate and adaptive arms of the immune system via their effects on endothelial function $(73,74)$. Cytotoxic molecules produced by innate immune cells including IL-17, ROS/ RNS, and IFNs all have detrimental effects on vascular function and excessive levels of these molecules in the systemic and reproductive vasculature would cause vasoconstriction and lead to organ dysfunction and damage (renal, placental, and possibly neural) (75-77). Activation of adaptive immunity by innate immune cells also occurs and numerous studies have shown that vasoactive molecules produced by $\mathrm{T}$ and $\mathrm{B}$ cells decrease endothelial function and induce PE-like features in animals (78-83). At the vascular level, endothelial cell injury due to excessive inflammation and oxidative stress leads to the production, secretion, and expression of cytokines including IL-6, chemokines such as chemokine ligand 5 (CCL5), and adhesion molecules such as VCAM, ICAM, and E-selectin. Additionally, studies in women with PE and animals with experimental PE consistently demonstrate that there is increased vascular endothelin-1 production and decreased nitric oxide bioavailability which likely results from the excessive inflammation mediated by immune system activation. More studies are needed to determine the molecular mechanisms by which innate immune system activation leads to endothelial dysfunction during pregnancy.

\section{Pathway Interplay and Therapeutic Targets}

We believe that each pathway is independent with respect to the initiating insult; however, subsequently there is considerable overlap between the pathways. For example, excessive innate immune system activity at the beginning of pregnancy would tend to inhibit trophoblast invasion, spiral artery remodeling, and fetal tolerance which would then exacerbate innate immune system activity. Likewise, pre-existing endothelial dysfunction would have similar effects on placental development leading to innate immunity and PE. Experimentally there is evidence that one can induce PE-like features in animals through any of the four pathways, including inducing endothelial dysfunction by inhibiting NOS/VEGF/TGF $\beta$ or high-fat feeding, inducing shallow trophoblast invasion and defective placentation, or excessively activating the maternal innate immune system via bacterial or viral mimetics. However, trying to identify the initial insult in women proves tougher. Current clinical testing and technologies likely miss the initial insult but rather examine the spectrum of downstream and convergent effects following innate immune system activation which differs between women. Improving endothelial function prior to pregnancy, early imaging of placental development, treatment of infections before pregnancy or early in pregnancy, and control of autoimmune diseases before and during pregnancy may all be beneficial in reducing the incidence and severity of $\mathrm{PE}$.

Given the potential central role of innate immunity in all four pathways leading to $\mathrm{PE}$, novel therapeutics targeting this arm of the immune system is needed. While current immunosuppressive therapies are not feasible for pregnant women, future research identifying the innate immune cells that are persistently activated in these pathways during pregnancy as well as unique characteristics of these activated cells would aid in the development of targeted therapies that eliminate just these specific cells and leave other innate immune cells alone.

\section{Conclusion}

Based on clinical and experimental evidence to date, we propose that $\mathrm{PE}$ develops through four independent pathways that either involve excessive innate immune system activation in response to abnormal physiology during pregnancy or excessive innate immunity before and/or during pregnancy. The excessive innate 
immunity and subsequent inflammation then converge, leading to angiogenic imbalance, endothelial/placental/renal dysfunction, and PE. The timing and severity of excessive innate immunity likely determine the onset and severity of PE. Studies that explore these proposed pathways in detail are needed to definitively determine

\section{References}

1. Bodnar LM, Catov JM, Klebanoff MA, Ness RB, Roberts JM. Prepregnancy body mass index and the occurrence of severe hypertensive disorders of pregnancy. Epidemiology (2007) 18(2):234-9. doi:10.1097/01.ede.0000254119.99660.e7

2. Stone JL, Lockwood CJ, Berkowitz GS, Alvarez M, Lapinski R, Berkowitz RL. Risk factors for severe preeclampsia. Obstet Gynecol (1994) 83(3):357-61.

3. Ros HS, Cnattingius S, Lipworth L. Comparison of risk factors for preeclampsia and gestational hypertension in a population-based cohort study. Am JEpidemiol (1998) 147(11):1062-70. doi:10.1093/oxfordjournals.aje.a009400

4. Bianco AT, Smilen SW, Davis Y, Lopez S, Lapinski R, Lockwood CJ. Pregnancy outcome and weight gain recommendations for the morbidly obese woman. Obstet Gynecol (1998) 91(1):97-102. doi:10.1016/S0029-7844(97)00578-4

5. Saftlas A, Wang W, Risch H, Woolson R, Hsu C, Bracken M. Prepregnancy body mass index and gestational weight gain as risk factors for preeclampsia and transient hypertension. Ann Epidemiol (2000) 10(7):475. doi:10.1016/ S1047-2797(00)00167-8

6. Li N, Liu E, Guo J, Pan L, Li B, Wang P, et al. Maternal prepregnancy body mass index and gestational weight gain on pregnancy outcomes. PLoS One (2013) 8(12):e82310. doi:10.1371/journal.pone.0082310

7. Mauricio MD, AldasoroM, OrtegaJ, VilaJM.Endothelial dysfunction in morbidobesity. Curr Pharm Des (2013) 19(32):5718-29. doi:10.2174/1381612811319320007

8. McCarthy FP, Kingdom JC, Kenny LC, Walsh SK. Animal models of preeclampsia; uses and limitations. Placenta (2011) 32(6):413-9. doi:10.1016/j. placenta.2011.03.010

9. Podjarny E, Baylis C, Losonczy G. Animal models of preeclampsia. Semin Perinatol (1999) 23(1):2-13. doi:10.1016/S0146-0005(99)80055-X

10. Masuyama H, Hiramatsu Y. Treatment with a constitutive androstane receptor ligand ameliorates the signs of preeclampsia in high-fat diet-induced obese pregnant mice. Mol Cell Endocrinol (2012) 348(1):120-7. doi:10.1016/j. mce.2011.07.047

11. Levine RJ, Lam C, Qian C, Yu KF, Maynard SE, Sachs BP, et al. Soluble endoglin and other circulating antiangiogenic factors in preeclampsia. N Engl J Med (2006) 355(10):992-1005. doi:10.1056/NEJMoa055352

12. Leik CE, Walsh SW. Neutrophils infiltrate resistance-sized vessels of subcutaneous fat in women with preeclampsia. Hypertension (2004) 44(1):72-7. doi:10.1161/01. HYP.0000130483.83154.37

13. Hubel CA, McLaughlin MK, Evans RW, Hauth BA, Sims CJ, Roberts JM. Fasting serum triglycerides, free fatty acids, and malondialdehyde are increased in preeclampsia, are positively correlated, and decrease within 48 hours post partum. Am JObstet Gynecol (1996) 174(3):975-82. doi:10.1016/S0002-9378(96)70336-8

14. Sattar N, Bendomir A, Berry C, Shepherd J, Greer IA, Packard CJ. Lipoprotein subfraction concentrations in preeclampsia: pathogenic parallels to atherosclerosis. Obstet Gynecol (1997) 89(3):403-8. doi:10.1016/S0029-7844(96)00514-5

15. Granger JP, Alexander BT, Llinas MT, Bennett WA, Khalil RA. Pathophysiology of hypertension during preeclampsia linking placental ischemia with endothelial dysfunction. Hypertension (2001) 38(3 Pt 2):718-22. doi:10.1161/01.HYP.38.3.718

16. Ding $X$, Yang Z, Han Y, Yu H. Fatty acid oxidation changes and the correlation with oxidative stress in different preeclampsia-like mouse models. PLoS One (2014) 9(10):e109554. doi:10.1371/journal.pone.0109554

17. Ding X, Yang Z, Han Y, Yu H. Adverse factors increase preeclampsia-like changes in pregnant mice with abnormal lipid metabolism. Chin Med J (Engl) (2014) 127(15):2814-8.

18. Ding X, Yang Z, Han Y, Yu H. Long-chain fatty acid oxidation changes in a beta2 glycoprotein I-induced preeclampsia-like mouse model. Placenta (2014) 35(6):392-7. doi:10.1016/j.placenta.2014.03.013

19. Xu XH, Shah PK, Faure E, Equils O, Thomas L, Fishbein MC, et al. Toll-like receptor- 4 is expressed by macrophages in murine and human lipid-rich atherosclerotic plaques and upregulated by oxidized LDL. Circulation (2001) 104(25):3103-8. doi:10.1161/hc5001.100631 the role of innate immune system activation under the various circumstances encountered during pregnancy. Ultimately, elimination of hyper-activated innate immune cells in $\mathrm{PE}$ may reduce the inflammation and endothelial dysfunction and improve maternal and fetal outcomes.

20. Tuten A, Aydemir B, Oncul M, Kiziler AR, Acikgoz AS, Korkmaz GG, et al. The association of lectin-like oxidized LDL receptor 1 (LOX-1) K167N and 3'UTR188CT polymorphisms with maternal plasma soluble LOX-1 levels and preeclampsia risk in Turkish population. Arch Gynecol Obstet (2015) 291(3):563-71. doi:10.1007/s00404-014-3457-4

21. Sankaralingam S, Xu H, Jiang Y, Sawamura T, Davidge ST. Evidence for increased methylglyoxal in the vasculature of women with preeclampsia: role in upregulation of LOX-1 and arginase. Hypertension (2009) 54(4):897-904. doi:10.1161/ HYPERTENSIONAHA.109.135228

22. Sankaralingam S, Xu Y, Sawamura T, Davidge ST. Increased lectin-like oxidized low-density lipoprotein receptor-1 expression in the maternal vasculature of women with preeclampsia: role for peroxynitrite. Hypertension (2009) 53(2):270-7. doi:10.1161/HYPERTENSIONAHA.108.122630

23. Zhang Y, Ye Y, Wang Y, Chen W. Inhibition of lectin-like oxidized low-density lipoprotein receptor 1 protects against plasma/hypoxia-mediated trophoblast dysfunction associated with preeclampsia. Gynecol Obstet Invest (2015) 79(2):90-6. doi:10.1159/000370195

24. Clausen TD, Mathiesen E, Ekbom P, Hellmuth E, Mandrup-Poulsen T, Damm P. Poor pregnancy outcome in women with type 2 diabetes. Diabetes Care (2005) 28(2):323-8. doi:10.2337/diacare.28.2.323

25. Garner PR, D'Alton ME, Dudley DK, Huard P, Hardie M. Preeclampsia in diabetic pregnancies. Am J Obstet Gynecol (1990) 163(2):505-8. doi:10.1016/0002-9378(90)91184-E

26. Cundy T, Slee F, Gamble G, Neale L. Hypertensive disorders of pregnancy in women with type 1 and type 2 diabetes. Diabet Med (2002) 19(6):482-9. doi:10.1046/j.1464-5491.2002.00729.x

27. Todorova K, Ivanov S, Zakharieva S, Batashki I. [Incidence of preeclampsia in pregnant women with type 1 diabetes mellitus]. Akush Ginekol (Sofiia) (2007) 46(4):15-9.

28. Hadi HA, Suwaidi JA. Endothelial dysfunction in diabetes mellitus. Vasc Health Risk Manag (2007) 3(6):853-76.

29. De Vriese AS, Verbeuren TJ, Van de Voorde J, Lameire NH, Vanhoutte PM. Endothelial dysfunction in diabetes. Br J Pharmacol (2000) 130(5):963-74. doi:10.1038/sj.bjp.0703393

30. Liu YJ, Saini A, Cohen DJ, Ooi BS. Modulation of macrophage proliferation by hyperglycemia. Mol Cell Endocrinol (1995) 114(1-2):187-92. doi:10.1016/0303-7207(95)96799-N

31. Faas MM, Spaans F, De Vos P. Monocytes and macrophages in pregnancy and pre-eclampsia. Front Immunol (2014) 5:298. doi:10.3389/fimmu.2014.00298

32. Duckitt K, Harrington D. Risk factors for pre-eclampsia at antenatal booking: systematic review of controlled studies. BMJ (2005) 330(7491):565. doi:10.1136/ bmj.38380.674340.E0

33. Bianco A, Stone J, Lynch L, Lapinski R, Berkowitz G, Berkowitz RL. Pregnancy outcome at age 40 and older. Obstet Gynecol (1996) 87(6):917-22. doi:10.1016/0029-7844(96)00045-2

34. Lee CJ, Hsieh TT, Chiu TH, Chen KC, Lo LM, Hung TH. Risk factors for pre-eclampsia in an Asian population. Int J Gynaecol Obstet (2000) 70(3):327-33. doi:10.1016/S0020-7292(00)00240-X

35. Dorjgochoo T, Gao YT, Chow WH, Shu XO, Yang G, Cai Q, et al. Obesity, age, and oxidative stress in middle-aged and older women. Antioxid Redox Signal (2011) 14(12):2453-60. doi:10.1089/ars.2010.3337

36. Csiszar A, Wang M, Lakatta EG, Ungvari Z. Inflammation and endothelial dysfunction during aging: role of NF-kappaB. J Appl Physiol (2008) 105(4):1333-41. doi:10.1152/japplphysiol.90470.2008

37. Sorescu D, Weiss D, Lassegue B, Clempus RE, Szocs K, Sorescu GP, et al. Superoxide production and expression of nox family proteins in human atherosclerosis. Circulation (2002) 105(12):1429-35. doi:10.1161/ 01.CIR.0000012917.74432.66

38. Hearps AC, Martin GE, Angelovich TA, Cheng WJ, Maisa A, Landay $\mathrm{AL}$, et al. Aging is associated with chronic innate immune activation and 
dysregulation of monocyte phenotype and function. Aging Cell (2012) 11(5):867-75. doi:10.1111/j.1474-9726.2012.00851.x

39. Bonney EA. Preeclampsia: a view through the danger model. J Reprod Immunol (2007) 76(1-2):68-74. doi:10.1016/j.jri.2007.03.006

40. Lyall F. Mechanisms regulating cytotrophoblast invasion in normal pregnancy and pre-eclampsia. Aust N Z J Obstet Gynaecol (2006) 46(4):266-73. doi:10.1111/j.1479-828X.2006.00589.x

41. Perez-Sepulveda A, Torres MJ, Khoury M, Illanes SE. Innate immune system and preeclampsia. Front Immunol (2014) 5:244. doi:10.3389/fimmu.2014.00244

42. Zhou Y, Damsky CH, Fisher SJ. Preeclampsia is associated with failure of human cytotrophoblasts to mimic a vascular adhesion phenotype. One cause of defective endovascular invasion in this syndrome?J Clin Invest (1997) 99(9):2152-64. doi:10.1172/JCI119388

43. Coukos G, Makrigiannakis A, Amin K, Albelda SM, Coutifaris C. Plateletendothelial cell adhesion molecule-1 is expressed by a subpopulation of human trophoblasts: a possible mechanism for trophoblast-endothelial interaction during haemochorial placentation. Mol Hum Reprod (1998) 4(4):357-67. doi:10.1093/ molehr/4.4.357

44. Chatterjee P, Weaver LE, Doersch KM, Kopriva SE, Chiasson VL, Allen SJ, et al. Placental toll-like receptor 3 and toll-like receptor $7 / 8$ activation contributes to preeclampsia in humans and mice. PLoS One (2012) 7(7):e41884. doi:10.1371/ journal.pone.0041884

45. Kim YM, Romero R, Oh SY, Kim CJ, Kilburn BA, Armant DR, et al. Toll-like receptor 4: a potential link between "danger signals," the innate immune system, and preeclampsia?Am J Obstet Gynecol (2005) 193(3 Pt 2):921-7. doi:10.1016/j. ajog.2005.07.076

46. Scharfe-Nugent A, Corr SC, Carpenter SB, Keogh L, Doyle B, Martin C, et al. TLR9 provokes inflammation in response to fetal DNA: mechanism for fetal loss in preterm birth and preeclampsia. J Immunol (2012) 188(11):5706-12. doi:10.4049/ jimmunol.1103454

47. Goulopoulou S, Matsumoto T, Bomfim GF, Webb RC. Toll-like receptor 9 activation: a novel mechanism linking placenta-derived mitochondrial DNA and vascular dysfunction in pre-eclampsia. Clin Sci (Lond) (2012) 123(7):429-35. doi:10.1042/CS20120130

48. Burton GJ, Woods AW, Jauniaux E, Kingdom JC. Rheological and physiological consequences of conversion of the maternal spiral arteries for uteroplacental blood flow during human pregnancy. Placenta (2009) 30(6):473-82. doi:10.1016/j. placenta.2009.02.009

49. Singh J, Ahmed A, Girardi G. Role of complement component C1q in the onset of preeclampsia in mice. Hypertension (2011) 58(4):716-24. doi:10.1161/ HYPERTENSIONAHA.111.175919

50. Rustveld LO, Kelsey SF, Sharma R. Association between maternal infections and preeclampsia: a systematic review of epidemiologic studies. Matern Child Health $J$ (2008) 12(2):223-42. doi:10.1007/s10995-007-0224-1

51. Lopez-Jaramillo P, Herrera JA, Arenas-Mantilla M, Jauregui IE, Mendoza MA. Subclinical infection as a cause of inflammation in preeclampsia. Am J Ther (2008) 15(4):373-6. doi:10.1097/MJT.0b013e318164c149

52. Conde-Agudelo A, Villar J, Lindheimer M. Maternal infection and risk of preeclampsia: systematic review and metaanalysis. Am J Obstet Gynecol (2008) 198(1):7-22. doi:10.1016/j.ajog.2007.07.040

53. Amarasekara R, Jayasekara RW, Senanayake H, Dissanayake VH. Microbiome of the placenta in pre-eclampsia supports the role of bacteria in the multifactorial cause of pre-eclampsia. J Obstet Gynaecol Res (2014). doi:10.1111/jog.12619

54. Kunnen A, Blaauw J, van Doormaal JJ, van Pampus MG, van der Schans CP, Aarnoudse JG, et al. Women with a recent history of early-onset pre-eclampsia have a worse periodontal condition. J Clin Periodontol (2007) 34(3):202-7. doi:10.1111/j.1600-051X.2006.01036.x

55. Zhang L, Yang H. Expression and localization of TLR4 and its negative regulator Tollip in the placenta of early-onset and late-onset preeclampsia. Hypertens Pregnancy (2012) 31(2):218-27. doi:10.3109/10641955.2011.642434

56. Bernardi FC, Felisberto F, Vuolo F, Petronilho F, Souza DR, Luciano TF, et al. Oxidative damage, inflammation, and toll-like receptor 4 pathway are increased in preeclamptic patients: a case-control study. Oxid Med Cell Longev (2012) 2012:636419. doi:10.1155/2012/636419

57. Faas MM, Schuiling GA, Baller JF, Visscher CA, Bakker WW. A new animal model for human preeclampsia: ultra-low-dose endotoxin infusion in pregnant rats. Am J Obstet Gynecol (1994) 171(1):158-64. doi:10.1016/0002-9378(94)90463-4

58. Chatterjee P, Chiasson VL, Kopriva SE, Young KJ, Chatterjee V, Jones KA, et al. Interleukin 10 deficiency exacerbates toll-like receptor 3-induced preeclampsia-like symptoms in mice. Hypertension (2011) 58(3):489-96. doi:10.1161/HYPERTENSIONAHA.111.172114

59. Tinsley JH, Chiasson VL, Mahajan A, Young KJ, Mitchell BM. Toll-like receptor 3 activation during pregnancy elicits preeclampsia-like symptoms in rats. Am J Hypertens (2009) 22(12):1314-9. doi:10.1038/ajh.2009.185

60. Tinsley JH, South S, Chiasson VL, Mitchell BM. Interleukin-10 reduces inflammation, endothelial dysfunction, and blood pressure in hypertensive pregnant rats. Am J Physiol Regul Integr Comp Physiol (2010) 298(3):R713-9. doi:10.1152/ ajpregu.00712.2009

61. Chatterjee P, Chiasson VL, Bounds KR, Mitchell BM. Regulation of the anti-inflammatory cytokines interleukin- 4 and interleukin- 10 during pregnancy. Front Immunol (2014) 5:253. doi:10.3389/fimmu.2014.00253

62. Chatterjee P, Kopriva SE, Chiasson VL, Young KJ, Tobin RP, Newell-Rogers K, et al. Interleukin-4 deficiency induces mild preeclampsia in mice. J Hypertens (2013) 31(7):1414-23. doi:10.1097/HJH.0b013e328360ae6c

63. Barnabe C, Faris PD, Quan H. Canadian pregnancy outcomes in rheumatoid arthritis and systemic lupus erythematosus. Int J Rheumatol (2011) 2011:345727. doi:10.1155/2011/345727

64. Salmon JE, Heuser C, Triebwasser M, Liszewski MK, Kavanagh D, Roumenina L, et al. Mutations in complement regulatory proteins predispose to preeclampsia: a genetic analysis of the PROMISSE cohort. PLoS Med (2011) 8(3):e1001013. doi:10.1371/journal.pmed.1001013

65. Tsai S, Hardison NE, James AH, Motsinger-Reif AA, Bischoff SR, Thames BH, et al. Transcriptional profiling of human placentas from pregnancies complicated by preeclampsia reveals disregulation of sialic acid acetylesterase and immune signalling pathways. Placenta (2011) 32(2):175-82. doi:10.1016/j.placenta.2010.11.014

66. Djurisic S, Hviid TV. HLA class Ib molecules and immune cells in pregnancy and preeclampsia. Front Immunol (2014) 5:652. doi:10.3389/fimmu.2014.00652

67. Martinez-Varea A, Pellicer B, Perales-Marin A, Pellicer A. Relationship between maternal immunological response during pregnancy and onset of preeclampsia. J Immunol Res (2014) 2014:210241. doi:10.1155/2014/210241

68. Hsu P, Santner-Nanan B, Dahlstrom JE, Fadia M, Chandra A, Peek M, et al. Altered decidual DC-SIGN+ antigen-presenting cells and impaired regulatory T-cell induction in preeclampsia. Am J Pathol (2012) 181(6):2149-60. doi:10.1016/j. ajpath.2012.08.032

69. Miko E, Szereday L, Barakonyi A, Jarkovich A, Varga P, Szekeres-Bartho J. Immunoactivation in preeclampsia: Vdelta2+ and regulatory $\mathrm{T}$ cells during the inflammatory stage of disease. J Reprod Immunol (2009) 80(1-2):100-8. doi:10.1016/j.jri.2009.01.003

70. Prins JR, Boelens HM, Heimweg J, Van der Heide S, Dubois AE, Van Oosterhout AJ, et al. Preeclampsia is associated with lower percentages of regulatory T cells in maternal blood. Hypertens Pregnancy (2009) 28(3):300-11. doi:10.1080/10641950802601237

71. Santner-Nanan B, Peek MJ, Khanam R, Richarts L, Zhu E, Fazekas de St Groth $B$, et al. Systemic increase in the ratio between Foxp3+ and IL-17-producing CD4+ T cells in healthy pregnancy but not in preeclampsia. J Immunol (2009) 183(11):7023-30. doi:10.4049/jimmunol.0901154

72. Toldi G, Svec P, Vasarhelyi B, Meszaros G, Rigo J, Tulassay T, et al. Decreased number of FoxP3+ regulatory T cells in preeclampsia. Acta Obstet Gynecol Scand (2008) 87(11):1229-33. doi:10.1080/00016340802389470

73. Roberts JM, Taylor RN, Musci TJ, Rodgers GM, Hubel CA, McLaughlin MK. Preeclampsia: an endothelial cell disorder. Am J Obstet Gynecol (1989) 161(5):1200-4. doi:10.1016/0002-9378(89)90665-0

74. Tinsley JH, Chiasson VL, South S, Mahajan A, Mitchell BM. Immunosuppression improves blood pressure and endothelial function in a rat model of pregnancy-induced hypertension. Am J Hypertens (2009) 22(10):1107-14. doi:10.1038/ ajh. 2009.125

75. Mitchell BM, Cook LG, Danchuk S, Puschett JB. Uncoupled endothelial nitric oxide synthase and oxidative stress in a rat model of pregnancy-induced hypertension. Am J Hypertens (2007) 20(12):1297-304. doi:10.1016/j.amjhyper.2007.08.007

76. Nguyen H, Chiasson VL, Chatterjee P, Kopriva SE, Young KJ, Mitchell BM. Interleukin-17 causes Rho-kinase-mediated endothelial dysfunction and hypertension. Cardiovasc Res (2013) 97(4):696-704. doi:10.1093/cvr/cvs422

77. Hubel CA. Oxidative stress in the pathogenesis of preeclampsia. Proc Soc Exp Biol Med (1999) 222(3):222-35. doi:10.1046/j.1525-1373.1999.d01-139.x

78. Zhou CC, Irani RA, Dai Y, Blackwell SC, Hicks MJ, Ramin SM, et al. Autoantibodymediated IL-6-dependent endothelin-1 elevation underlies pathogenesis in a mouse model of preeclampsia. J Immunol (2011) 186(10):6024-34. doi:10.4049/ jimmunol.1004026 
79. Zhou CC, Irani RA, Zhang Y, Blackwell SC, Mi T, Wen J, et al. Angiotensin receptor agonistic autoantibody-mediated tumor necrosis factor-alpha induction contributes to increased soluble endoglin production in preeclampsia. Circulation (2010) 121(3):436-44. doi:10.1161/CIRCULATIONAHA.109.902890

80. Zhou CC, Zhang Y, Irani RA, Zhang H, Mi T, Popek EJ, et al. Angiotensin receptor agonistic autoantibodies induce pre-eclampsia in pregnant mice. Nat Med (2008) 14(8):855-62. doi:10.1038/nm.1856

81. Lamarca B. The role of immune activation in contributing to vascular dysfunction and the pathophysiology of hypertension during preeclampsia. Minerva Ginecol (2010) 62(2):105-20.

82. LaMarca B, Parrish M, Ray LF, Murphy SR, Roberts L, Glover P, et al. Hypertension in response to autoantibodies to the angiotensin II type I receptor (AT1-AA) in pregnant rats: role of endothelin-1. Hypertension (2009) 54(4):905-9. doi:10.1161/ HYPERTENSIONAHA.109.137935
83. LaMarca B, Wallace K, Herse F, Wallukat G, Martin JN Jr, Weimer A, et al. Hypertension in response to placental ischemia during pregnancy: role of B lymphocytes. Hypertension (2011) 57(4):865-71. doi:10.1161/ HYPERTENSIONAHA.110.167569

Conflict of Interest Statement: M. Karen Newell-Rogers and Brett M. Mitchell have a relationship with VG Life Sciences Inc. Kelsey R. Bounds has no conflict of interests to declare.

Copyright (C) 2015 Bounds, Newell-Rogers and Mitchell. This is an open-access article distributed under the terms of the Creative Commons Attribution License (CC BY). The use, distribution or reproduction in other forums is permitted, provided the original author(s) or licensor are credited and that the original publication in this journal is cited, in accordance with accepted academic practice. No use, distribution or reproduction is permitted which does not comply with these terms. 